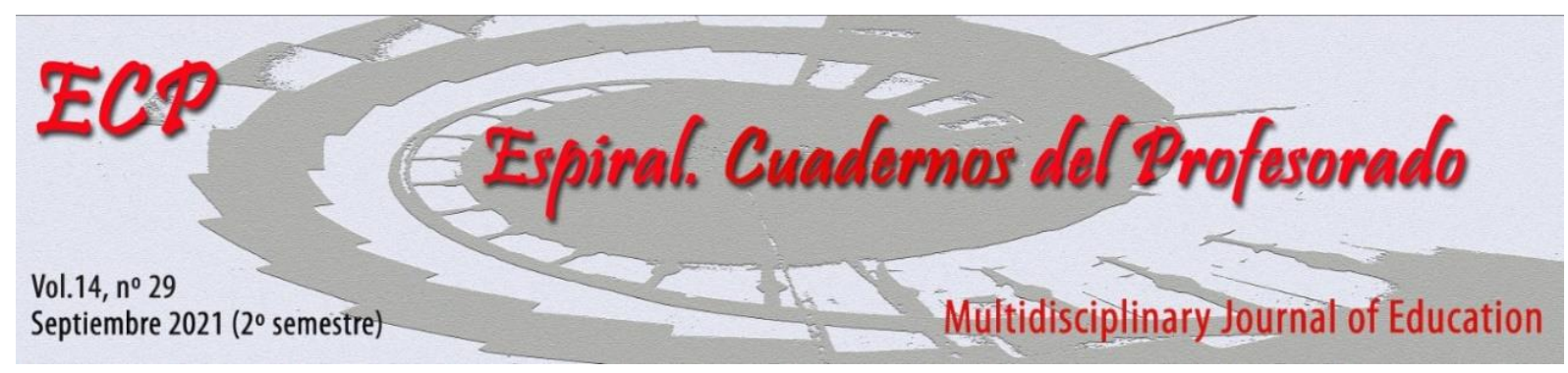

\title{
Síndrome de Burnout en docentes universitarios latinoamericanos: Una revisión sistemática
}

\section{Burnout sindrome in Latin-American higher education professors: A systematic review}

\author{
José Luis Rojas-Solís ${ }^{1}$, Bianca Areli Totolhua-Reyes ${ }^{1}$, y Diana Jezabel Rodríguez-Vásquez ${ }^{1}$ \\ Facultad de Psicología, Benemérita Universidad Autónoma de Puebla, México
}

\begin{abstract}
Resumen
El estudio del burnout en docentes universitarios ha cobrado relevancia últimamente en la comunidad científica debido a los efectos indeseables del estrés. Por ello, este trabajo tiene como objetivo realizar una revisión sistemática exploratoria sobre el burnout en docentes latinoamericanos, con la finalidad de determinar y contrastar elementos metodológicos y hallazgos principales de las investigaciones más relevantes publicadas entre 1994 y 2019. Siguiendo los lineamientos del modelo PRISMA, se identificaron un total de 25 artículos de investigación provenientes de nueve bases de datos electrónicas (APA PsycNet, EBSCO, LA Referencia, SAGE Journals, Scielo, Science Direct, Scopus, Taylor \& Francis y Web of Science). Entre los principales resultados destaca el uso del enfoque cuantitativo, así como del Maslach Burnout Inventory (MBI) en la recolección de datos; así mismo se encontró una ausencia significativa en la implementación de algunos aspectos éticos y legales en el procedimiento de los estudios evaluados. Finalmente, se concluye que el burnout es un fenómeno presente en los y las docentes de educación superior y se asocia con diversos factores psicosociales y organizacionales. Entre las posibles implicaciones destaca la necesidad de mayores esfuerzos en la prevención de este fenómeno, a través del estudio de los factores protectores.
\end{abstract}

Palabras clave: Burnout; Docente; Universidad; Revisión sistemática.

\begin{abstract}
Research about burnout in university professors has recently gained relevance in the scientific community due to the undesirable effects of stress. Therefore, this work aims to carry out an exploratory systematic review on burnout in Latin American professors, in order to determine and contrast methodological elements and main findings of the most relevant research published between 1994 and 2019. Throughout the guidelines of the PRISMA model, a total of 25 research articles were identified from nine electronic databases (APA PsycNet, EBSCO, LA Reference, SAGE Journals, Scielo, Science Direct, Scopus, Taylor \& Francis and Web of Science). The main results show the use of the quantitative approach, as well as the Maslach Burnout Inventory (MBI) in data collection; besides, a significant absence was found in the implementation of some ethical and legal aspects in the procedure of the evaluated studies. Finally, it is concluded that burnout is a phenomenon present in higher education professors and is associated with various psychosocial and organizational factors. Among the possible implications, the need for greater efforts in the prevention of this phenomenon, through the study of protective factors, was pointed out.
\end{abstract}

Keywords: Burnout; Professor; Higher education; Systematic review.

Fecha de recepción: 25/02/2021

Fecha de aceptación: 22/06/2021

José Luis Rojas-Solís, Benemérita Universidad Autónoma de Puebla, México Correspondencia: jlrojassolis@gmail.com 


\section{Introducción}

Debido a la preocupación por el desarrollo y la mejora en la educación superior se ha evidenciado un interés por el trabajo docente, sobre todo considerando las responsabilidades y el compromiso que se les exige. Tal vez por ello, esta profesión se ha considerado como una actividad estresante que trae consigo repercusiones en la salud mental, física y en el desempeño profesional de los profesores quienes a su vez corren un alto riesgo de desarrollar síndrome de burnout (SB), el cual es un fenómeno social que expresa crisis dentro del desempeño laboral, de modo especial en aquellos que están en contacto con otros, tal como sucede en la práctica educativa (Jeunon et al., 2017).

Una de las definiciones históricamente más difundidas del SB es la que Freudenberguer, en su papel de autor pionero, desde una perspectiva clínica lo explica como un estado de deterioro de energía, de pérdida de motivación y de compromiso, que va acompañado de un amplio abanico de síntomas físicos y mentales (Juárez-García et al., 2014), por otra parte, durante las últimas dos décadas la definición propuesta por Maslach y Jackson (1986) ha obtenido primacía dentro de la literatura científica, en ella afirman que es una manifestación comportamental derivada del estrés laboral y lo entienden como un síndrome tridimensional caracterizado por cansancio emocional, despersonalización en el trato con clientes y usuarios y la realización personal.

Ahora bien, el fenómeno ha ido en aumento y con ello sus consecuencias en el personal docente universitario, quienes por la naturaleza de su trabajo se encuentran expuestos a exigencias psicológicas, provocadas por la cantidad y la diversidad de tareas que deben desempeñar (Pujol-Cols et al., 2019), es así como las respuestas que ellos dan ante esta situación son la insatisfacción laboral y profesional, los intentos de abandono, la reducción en la productividad, la pérdida de compromiso, el interés y otras repercusiones relacionadas con su salud (Lackritz, 2004). Del mismo modo, en las políticas educativas que se implementan en los diferentes países se encuentran inmersos factores psicosociales y de riesgo relacionados con el $\mathrm{SB}$, que se ven reforzados por las presiones de diversa índole como la constante interacción con alumnos o las exigencias laborales propias de cada institución, lo cual ha provocado el incremento del desgaste psíquico y la disminución del desempeño laboral (Villamar et al., 2019), es así como la calidad educativa y su compromiso en la práctica de la formación social y cultural se ven perjudicados (Palmer et al., 2016).

Por lo anterior, el presente estudio pretende explorar las características metodológicas, los instrumentos, los procedimientos de recolección y los aspectos éticos de la investigación existente sobre SB en docentes universitarios de Latinoamérica. El objetivo ulterior de esta revisión sistemática exploratoria es contribuir a la literatura mediante la síntesis y difusión de los hallazgos, para ello las siguientes preguntas servirían de guía:

- ¿Cuáles son las principales características bibliográficas de las investigaciones?

- ¿Cuál es la terminología ligada al SB?

- ¿Qué tipos de estudios abordan e informan acerca del SB y cuáles son sus principales características metodológicas?

- ¿Qué características poseen las muestras de estudio?

- ¿Cuál fue el procedimiento de recolección y los aspectos éticos?

- ¿Cuáles son los instrumentos de medida utilizados en las investigaciones?

\section{Método}

\section{Diseño}

La búsqueda sistemática de la literatura se enmarca en el modelo de investigación teórica, con un alcance exploratorio (Fernández-Sánchez et al., 2020) y descriptivo siguiendo el modelo PRISMA, el cual provee de un marco robusto y comprensivo para la evaluación de los artículos incluidos en la revisión (Moher et al., 2009). Es preciso aclarar que esta revisión exploratoria se encamina a contestar las preguntas previamente planteadas por lo que no fue posible implementar la herramienta PICOS, en relación con participantes, intervenciones, comparaciones, resultados y diseño de los estudios (Urrutia y Bonfill, 2010).

Espiral. Cuadernos del Profesorado | ISSN 1988-7701 | 2021, 14(29), 136-150 
La búsqueda electrónica se realizó a través bases de datos, relevantes en investigación: Apa PsycNet, EBSCO, LA Referencia, SAGE Journals, Scielo, Science Direct, Scopus, Taylor \& Francis y Web Of Science; para ello se utilizaron los términos en inglés "burnout", "professors" y "higher education" mientras que en la traducción al español se utilizó "burnout", "docentes" y "universidad", conformando las siguientes cadenas de búsqueda: "burnout AND professors AND higher education" y "burnout AND docentes AND universidad", el propósito del operador booleano AND fue aportar especificidad a la estrategia de búsqueda. El proceso se realizó en el periodo comprendido entre los meses de julio y agosto del 2020.

Los criterios de inclusión fueron: estudios publicados entre 1994 a 2019, en inglés o español con muestra conformada por docentes de educación superior pertenecientes a países de Latinoamérica y el acceso a los textos debió ser gratuito.

Los resultados se registraron en una hoja de cálculo de Microsoft (C) Excel 2018 y se eligieron mediante un proceso dividido en cuatro fases (ver Figura 1).

\section{Figura 1}

Diagrama de Flujo (Fuente: Elaboración propia).

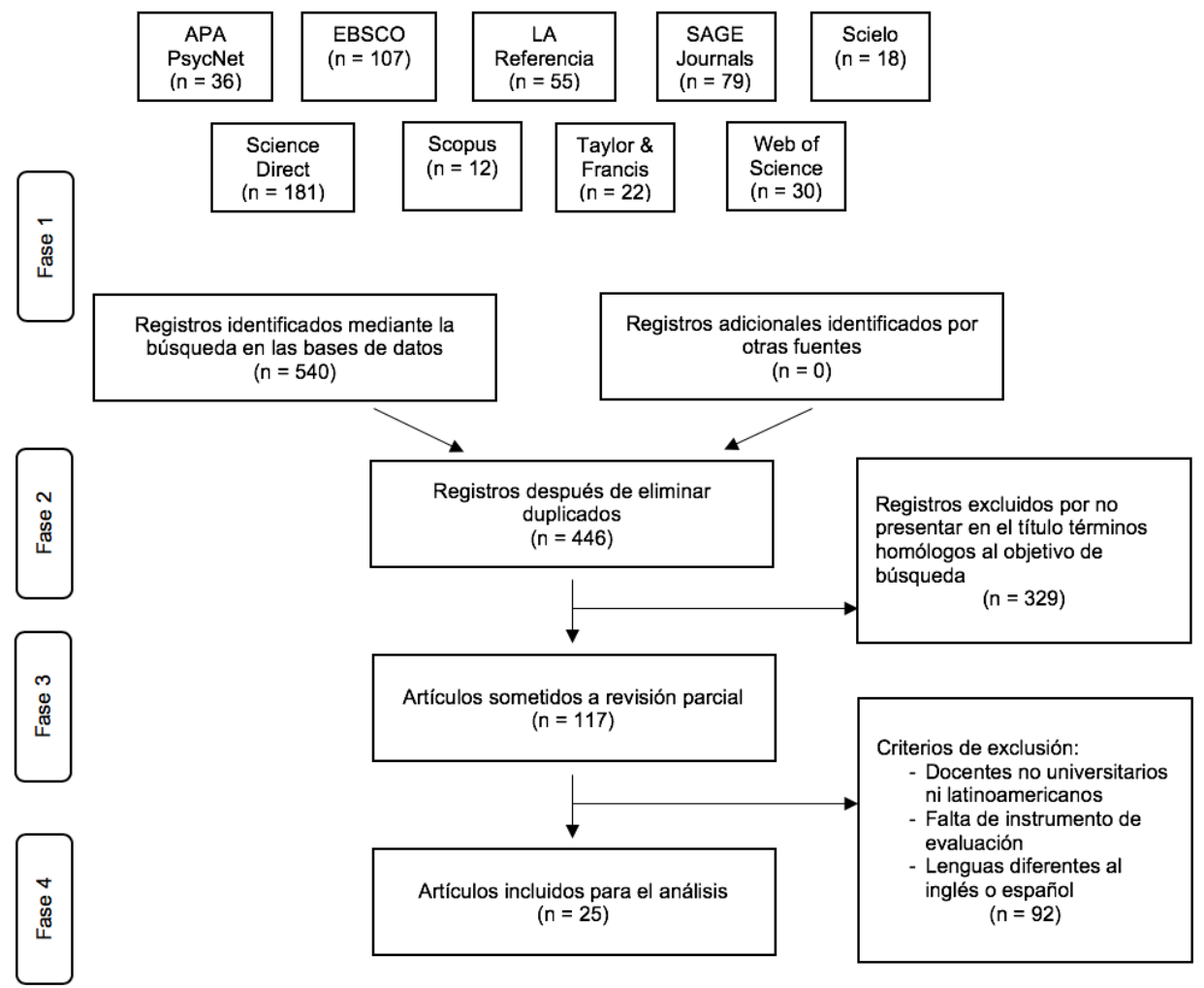

\section{Consideraciones éticas}

La participación de seres humanos y el riesgo asociado a ello fue inexistente, no obstante, se han seguido las directrices éticas de la American Psychological Association (2017) para este tipo de estudios, en especial el reconocimiento de autoría de las obras consultadas a través de las citas y referencias respectivas.

\section{Resultados}

Con respecto a las principales características bibliográficas de las investigaciones, el rango de publicación se encuentra entre 2005-2019, los 25 documentos se publicaron en revistas especializadas en 
el área de la salud y, en su mayoría, escritos en español. Dentro de los objetivos resalta la búsqueda de la prevalencia, la determinación de los niveles de afectación y la asociación con distintos factores.

En cuanto a la terminología ligada al SB, existió una variedad de palabras clave, en inglés y español, utilizadas para nombrar al SB, sin embargo, siete se utilizaron con más frecuencia (ver Figura 2).

\section{Figura 2}

Palabras clave (Fuente: Elaboración propia).

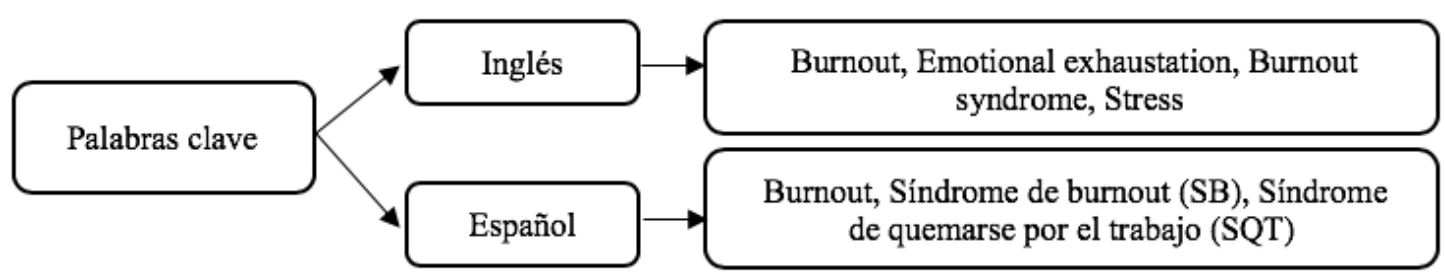

Con relación a las principales características metodológicas, la mayoría utilizó un enfoque cuantitativo con alcances descriptivos y correlacionales; todos los estudios tuvieron diseños no experimentales y transversales (ver Tabla 1).

Tabla 1

Características metodológicas de los estudios

\begin{tabular}{|c|c|c|c|c|}
\hline Autoría, año & Enfoque & Alcance & Diseño & $\begin{array}{c}\text { Propósito (Analizar, identificar, evaluar, } \\
\text { determinar, explorar) }\end{array}$ \\
\hline $\begin{array}{l}\text { García-Arroyo \& } \\
\text { Osca (2019) }\end{array}$ & $\mathrm{Ct}$ & $\mathrm{C}$ & NExpl, T & $\begin{array}{l}\text { La relación entre el estrés por sobrecarga de } \\
\text { trabajo y agotamiento emocional }\end{array}$ \\
\hline Romero (2019) & $\mathrm{Ct}$ & $\mathrm{D}$ & NExpl, T & $\begin{array}{l}\text { La configuración del sentido de la profesión } \\
\text { académica y condiciones laborales }\end{array}$ \\
\hline Villamar et al. (2019) & $\mathrm{Ct}$ & $\mathrm{C}$ & NExpl, T & $\begin{array}{l}\text { La relación entre factores psicosociales del } \\
\text { trabajo académico y SB }\end{array}$ \\
\hline $\begin{array}{l}\text { Mesurado \& Laudadío } \\
\text { (2019) }\end{array}$ & $\mathrm{Ct}$ & $\mathrm{C}$ & NExpl, T & $\begin{array}{l}\text { La relación entre nivel de experiencia, capital } \\
\text { psicológico y engagement con SB }\end{array}$ \\
\hline Alves (2019) & $\mathrm{Ct}$ & $\mathrm{D}$ & NExpl, T & El impacto del SB en la calidad de vida \\
\hline $\begin{array}{l}\text { Alpízar \& Araya } \\
\quad(2018)\end{array}$ & $\mathrm{Ct}$ & $\mathrm{D}$ & NExpl, T & $\begin{array}{l}\text { La incidencia del SB y caracterizar las clases } \\
\text { latentes según variables sociodemográficas }\end{array}$ \\
\hline $\begin{array}{l}\text { Rodríguez \& Sánchez } \\
\qquad(2018)\end{array}$ & $\mathrm{Ct}$ & $\mathrm{D}$ & NExpl, T & $\begin{array}{l}\text { Las diferencias entre los componentes del SB } \\
\text { según variables sociodemográficas }\end{array}$ \\
\hline Brito (2018) & $\mathrm{Ct}$ & $\mathrm{D}$ & NExpl, T & La propensión a padecer SB \\
\hline $\begin{array}{l}\text { García-Arroyo \& } \\
\text { Osca }(2017)\end{array}$ & $\mathrm{Ct}$ & $\mathrm{E}, \mathrm{C}$ & NExpl, T & $\begin{array}{l}\text { La relación entre afrontamiento centrado en la } \\
\text { acción y de la emoción con las dimensiones del } \\
\text { SB }\end{array}$ \\
\hline García et al. (2017) & $\mathrm{Ct}$ & $\mathrm{D}, \mathrm{C}$ & NExpl, T & $\begin{array}{l}\text { La relación entre SB y sentimientos de } \\
\text { autoeficacia con el rendimiento académico }\end{array}$ \\
\hline Jeunon et al. (2017) & Mix & $\mathrm{D}$ & NExpl, T & $\begin{array}{l}\text { La prevalencia de SB y factores de riesgo } \\
\text { asociados }\end{array}$ \\
\hline Cabezas (2016) & $\mathrm{Ct}$ & $\mathrm{D}$ & NExpl, T & $\begin{array}{l}\text { Los diversos factores incluyendo actividades } \\
\text { docentes, funciones administrativas, } \\
\text { características y condiciones de tipo laboral y } \\
\text { su incidencia }\end{array}$ \\
\hline $\begin{array}{l}\text { Bustamante et al. } \\
\text { (2016) }\end{array}$ & $\mathrm{Ct}$ & $\mathrm{D}$, & NExpl, T & Los niveles de SB existentes \\
\hline
\end{tabular}

Espiral. Cuadernos del Profesorado | ISSN 1988-7701 | 2021, 14(29), 136-150 


\begin{tabular}{|c|c|c|c|c|}
\hline Ilaja \& Reyes (2016) & $\mathrm{Ct}$ & $\mathrm{D}, \mathrm{C}$ & NExpl, T & $\begin{array}{l}\text { Los aspectos relacionados con el SB en las } \\
\text { distintas tareas realizadas por los docentes }\end{array}$ \\
\hline Chavarría et al. (2016) & $\mathrm{Ct}$ & $\mathrm{D}$ & NExpl, T & La presencia de SB y factores asociados \\
\hline Ruiz et al. (2015) & $\mathrm{Ct}$ & $\mathrm{D}, \mathrm{C}$ & NExpl, T & $\begin{array}{l}\text { La presencia del SB y su relación con variables } \\
\text { del contexto laboral }\end{array}$ \\
\hline Cárdenas et al. (2014) & $\mathrm{Ct}$ & $\mathrm{C}$ & NExpl, T & $\begin{array}{l}\text { La relación entre los niveles de estrés percibido } \\
\text { y SB }\end{array}$ \\
\hline Gaxiola-Villa (2014) & $\mathrm{Ct}$ & $\mathrm{D}, \mathrm{C}$ & NExpl, T & $\begin{array}{c}\text { La relación entre bienestar psicológico y } \\
\text { desgaste profesional }\end{array}$ \\
\hline Zárate \& Arita (2013) & $\mathrm{Ct}$ & $\mathrm{D}, \mathrm{C}$ & NExpl, T & $\begin{array}{l}\text { Las condiciones laborales predisponentes del } \\
\text { SB }\end{array}$ \\
\hline Borges et al. (2012) & $\mathrm{Ct}$ & $\mathrm{D}$ & NExpl, T & $\begin{array}{l}\text { La prevalencia del SB en relación con } \\
\text { condiciones sociolaborales }\end{array}$ \\
\hline $\begin{array}{l}\text { Rojas \& Grisales } \\
\text { (2011) }\end{array}$ & $\mathrm{Ct}$ & $\mathrm{D}$ & NExpl, T & $\begin{array}{l}\text { La prevalencia del SB y su relación con el tipo } \\
\text { de vinculación laboral }\end{array}$ \\
\hline Ferrel et al. (2010) & $\mathrm{Ct}$ & $\mathrm{D}$ & NExpl, T & $\begin{array}{l}\text { La presencia o ausencia SB teniendo en cuenta } \\
\text { edad y tipo de vinculación }\end{array}$ \\
\hline Caballero et al. (2009) & $\mathrm{Ct}$ & $\mathrm{D}, \mathrm{C}$ & NExpl, T & La prevalencia e incidencia del SB \\
\hline Rojas et al. (2009) & $\mathrm{Ct}$ & $\mathrm{E}, \mathrm{D}$ & NExpl, T & $\begin{array}{c}\text { La prevalencia del SB, su relación con diversas } \\
\text { variables demográficas, laborales y satisfacción } \\
\text { laboral }\end{array}$ \\
\hline Ponce et al. (2005) & Mix & $\mathrm{E}, \mathrm{D}$ & NExpl, T & $\begin{array}{c}\text { La presencia del SB por estrés laboral } \\
\text { asistencial }\end{array}$ \\
\hline
\end{tabular}

Nota: N/E: No Especificado, Ct: Cuantitativo, Mix: Mixto, D: Descriptivo, C: Correlacional, E: Exploratorio, NExpl: No Experimental, T:Transversal, SB: Síndrome de Burnout.

Concerniente a las características de las muestras de estudio, en su mayoría provienen de Colombia, México y Ecuador. La edad de los participantes osciló entre los 20 y 71 años, los grupos de estudio incluyeron participantes de ambos sexos y el proceso de selección predominante fue no probabilístico (72\%). En cuanto a la prevalencia del fenómeno, 60\% de las investigaciones destacó niveles medio-bajos de SB en los docentes, mientras que un 20\% señaló niveles altos y significativos (ver Tabla 2).

\section{Tabla 2}

Características de los grupos de estudio.

\begin{tabular}{|c|c|c|c|c|}
\hline $\begin{array}{l}\text { Autoría, } \\
\text { año }\end{array}$ & $\begin{array}{c}\text { Tamaño/Tipo/Rango de } \\
\text { edad/ Muestreo }\end{array}$ & Sexo & País & Prevalencia \\
\hline $\begin{array}{c}\text { García- } \\
\text { Arroyo \& } \\
\text { Osca (2019) }\end{array}$ & $\begin{array}{c}202 \text { docentes } \\
\text { N/E } \\
\text { NP }\end{array}$ & $\begin{array}{c}73 \% \\
\text { hombres } \\
27 \% \text { mujeres }\end{array}$ & Ecuador & $\mathrm{N} / \mathrm{E}$ \\
\hline $\begin{array}{l}\text { Romero, } \\
2019\end{array}$ & $\begin{array}{l}\text { NP } \\
160 \text { docentes } \\
23-65 \text { años } \\
\text { NP }\end{array}$ & $\begin{array}{l}78 \text { hombres } \\
82 \text { mujeres }\end{array}$ & Colombia & $\begin{array}{c}\text { Evidencia puntajes altos en las } \\
\text { dimensiones de cinismo y } \\
\text { agotamiento }\end{array}$ \\
\hline $\begin{array}{l}\text { Villamar et } \\
\text { al. (2019) }\end{array}$ & $\begin{array}{l}247 \text { docentes } \\
\text { N/E } \\
\text { NP }\end{array}$ & $\begin{array}{l}122 \text { hombres } \\
125 \text { mujeres }\end{array}$ & México & $\begin{array}{c}\text { Nivel alto }(19.8 \%) \\
\text { Nivel crítico }(13.8 \%)\end{array}$ \\
\hline $\begin{array}{l}\text { Mesurado } \\
\text { \& Laudadío } \\
\text { (2019) }\end{array}$ & $\begin{array}{l}250 \text { docentes } \\
\text { N/E } \\
\text { NP }\end{array}$ & $\begin{array}{c}51 \% \\
\text { hombres } \\
49 \% \text { mujeres }\end{array}$ & Argentina & $\mathrm{N} / \mathrm{E}$ \\
\hline Alves, 2019 & $\begin{array}{l}366 \text { docentes } \\
\text { 29-69 años } \\
\text { NP }\end{array}$ & $\begin{array}{l}207 \text { hombres } \\
139 \text { mujeres }\end{array}$ & Brasil & $\mathrm{El} 36.6 \%$ presentaron agotamiento \\
\hline
\end{tabular}




\begin{tabular}{|c|c|c|c|c|}
\hline $\begin{array}{l}\text { Alpízar \& } \\
\text { Araya } \\
(2018)\end{array}$ & $\begin{array}{l}251 \text { docentes } \\
\text { N/E } \\
\text { PRO }\end{array}$ & $\mathrm{N} / \mathrm{E}$ & Costa Rica & $\begin{array}{c}\text { Se confirma la existencia presencia } \\
\text { de SB en porcentajes bajos y } \\
\text { medios }\end{array}$ \\
\hline $\begin{array}{l}\text { Rodríguez } \\
\text { \& Sánchez } \\
\text { (2018) }\end{array}$ & $\begin{array}{l}260 \text { docentes } \\
20-61 \text { años } \\
\text { NP }\end{array}$ & $\begin{array}{l}147 \text { hombres } \\
113 \text { mujeres }\end{array}$ & Perú & $\begin{array}{l}\text { Existen un nivel medio de SB } \\
\text { según el análisis de cada uno de } \\
\text { sus componentes. }\end{array}$ \\
\hline Brito, 2018 & $\begin{array}{l}20 \text { docentes } \\
\text { N/E } \\
\text { NP }\end{array}$ & N/E & México & $\begin{array}{l}\text { Se evidencia un grado de } \\
\text { agotamiento significativo, niveles } \\
\text { altos de cinismo y bajos de } \\
\text { eficacia profesional }\end{array}$ \\
\hline $\begin{array}{l}\text { García- } \\
\text { Arroyo \& }\end{array}$ & $\begin{array}{l}202 \text { docentes } \\
\text { N/E }\end{array}$ & $\begin{array}{l}73 \% \\
\text { hombres }\end{array}$ & Ecuador & 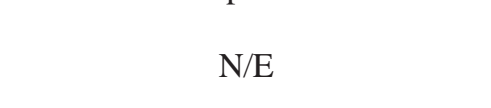 \\
\hline Osca (2017) & NP & $\begin{array}{c}27 \% \text { mujeres } \\
41.7 \%\end{array}$ & & \\
\hline $\begin{array}{l}\text { García et al. } \\
\text { (2017) }\end{array}$ & $\begin{array}{l}37 \text { docentes } \\
\leq 20>60 \\
\mathrm{NP}\end{array}$ & $\begin{array}{l}\text { hombres } \\
58.3 \% \\
\text { mujeres }\end{array}$ & Colombia & $\mathrm{N} / \mathrm{E}$ \\
\hline $\begin{array}{l}\text { Jeunon et } \\
\text { al. }(2017)\end{array}$ & $\begin{array}{l}309 \text { docentes } \\
\text { N/E } \\
\text { NP }\end{array}$ & $\begin{array}{l}210 \text { hombres } \\
99 \text { mujeres }\end{array}$ & Brasil & $\begin{array}{c}\text { Baja prevalencia en las } \\
\text { dimensiones de agotamiento } \\
\text { emocional y despersonalización }\end{array}$ \\
\hline $\begin{array}{l}\text { Cabezas, } \\
2016\end{array}$ & $\begin{array}{l}52 \text { docentes } \\
\mathrm{N} / \mathrm{E} \\
\mathrm{NP}\end{array}$ & $\begin{array}{l}31 \text { hombres } \\
21 \text { mujeres }\end{array}$ & Ecuador & $\mathrm{N} / \mathrm{E}$ \\
\hline $\begin{array}{l}\text { Bustamante } \\
\text { et al. (2016) }\end{array}$ & $\begin{array}{l}45 \text { docentes } \\
\text { N/E } \\
\text { NP }\end{array}$ & $\begin{array}{c}16 \% \\
\text { hombres } \\
84 \% \text { mujeres }\end{array}$ & Venezuela & $\begin{array}{c}\text { El } 16 \% \text { de los docentes presenta el } \\
\text { SB en niveles altos }\end{array}$ \\
\hline $\begin{array}{l}\text { Ilaja \& } \\
\text { Reyes } \\
(2016)\end{array}$ & $\begin{array}{l}60 \text { docentes } \\
\leq 30>60 \\
\mathrm{NP}\end{array}$ & $\begin{array}{l}35 \text { hombres } \\
25 \text { mujeres }\end{array}$ & Ecuador & $\mathrm{N} / \mathrm{E}$ \\
\hline $\begin{array}{l}\text { Chavarría et } \\
\text { al. (2016) }\end{array}$ & $\begin{array}{l}36 \text { docentes } \\
30-60 \text { años } \\
\text { NP }\end{array}$ & $\begin{array}{l}66.66 \% \\
\text { hombres } \\
33.34 \% \\
\text { mujeres }\end{array}$ & México & $\begin{array}{l}\text { EL } 53.33 \% \text { de los docentes se } \\
\text { ubicaron con nivel alto de SB }\end{array}$ \\
\hline $\begin{array}{l}\text { Ruiz et al. } \\
\text { (2015) }\end{array}$ & $\begin{array}{l}30 \text { docentes } \\
20-60 \text { años } \\
\text { NP }\end{array}$ & $\begin{array}{l}3 \text { hombres } 27 \\
\text { mujeres }\end{array}$ & Colombia & $\begin{array}{c}\text { Cansancio emocional bajo } \\
(66.66 \%) \\
\text { Nivel bajo de despersonalización } \\
(96.66 \%) \\
\text { Realización personal }(80 \%)\end{array}$ \\
\hline $\begin{array}{l}\text { Cárdenas et } \\
\text { al. (2014) }\end{array}$ & $\begin{array}{l}59 \text { docentes } \\
\text { N/E } \\
\text { NP }\end{array}$ & $\begin{array}{l}32.2 \% \\
\text { hombres } \\
67.8 \% \\
\text { mujeres }\end{array}$ & México & N/E \\
\hline $\begin{array}{l}\text { Gaxiola- } \\
\text { Villa, } 2014\end{array}$ & $\begin{array}{l}120 \text { docentes } \\
\text { N/E } \\
\text { NP }\end{array}$ & 120 mujeres & México & $\mathrm{N} / \mathrm{E}$ \\
\hline $\begin{array}{c}\text { Zárate \& } \\
\text { Arita (2013) }\end{array}$ & $\begin{array}{l}23 \text { docentes } \\
\text { N/E } \\
\mathrm{NP}\end{array}$ & $\mathrm{N} / \mathrm{E}$ & México & $\begin{array}{c}\text { Baja ilusión por el trabajo } \\
\quad(26.08 \%) \\
\text { Desgaste psíquico }(20.08 \%) \\
\text { Desencanto profesional }(13.04 \%)\end{array}$ \\
\hline $\begin{array}{l}\text { Borges et } \\
\text { al. }(2012)\end{array}$ & $\begin{array}{l}145 \text { docentes } \\
\leq 35>60 \\
\mathrm{NP}\end{array}$ & $\begin{array}{l}42.1 \% \\
\text { hombres } \\
57.9 \% \\
\text { mujeres }\end{array}$ & Venezuela & La prevalencia del SN fue del $21 \%$ \\
\hline
\end{tabular}

Espiral. Cuadernos del Profesorado | ISSN 1988-7701 | 2021, 14(29), 136-150 


\begin{tabular}{|c|c|c|c|c|}
\hline $\begin{array}{c}\text { Rojas \& } \\
\text { Grisales } \\
(2011)\end{array}$ & $\begin{array}{c}89 \text { docentes } \\
\text { N/E } \\
\mathrm{NP}\end{array}$ & N/E & Colombia & $\begin{array}{l}\text { Casos probables: } 19.1 \% \\
\text { Casos en riesgo: } 49.9 \%\end{array}$ \\
\hline $\begin{array}{l}\text { Ferrel et al. } \\
\qquad(2010)\end{array}$ & $\begin{array}{c}225 \text { docentes } \\
27-56 \text { años } \\
\text { NP }\end{array}$ & N/E & Colombia & $\begin{array}{c}\text { El 30\% de la muestra presenta } \\
\text { desgaste emocional }\end{array}$ \\
\hline $\begin{array}{l}\text { Caballero et } \\
\text { al. (2009) }\end{array}$ & $\begin{array}{c}101 \text { docentes } \\
23-65 \text { años } \\
\text { PRO }\end{array}$ & $\begin{array}{c}38,6 \% \\
\text { hombres } \\
61,4 \% \\
\text { mujeres }\end{array}$ & Colombia & $\begin{array}{l}\text { Prevalencia del SB en el } 10 \% \text { de la } \\
\text { muestra }\end{array}$ \\
\hline $\begin{array}{l}\text { Rojas et al. } \\
\quad(2009)\end{array}$ & $\begin{array}{c}89 \text { docentes } \\
\text { N/E } \\
\text { PRO }\end{array}$ & N/E & Colombia & $\begin{array}{l}\text { Casos confirmados } 19,1 \% \\
\text { En riesgo de sufrirlo } 49.4 \%\end{array}$ \\
\hline $\begin{array}{l}\text { Ponce et al. } \\
\qquad(2005)\end{array}$ & $\begin{array}{c}274 \text { docentes } \\
23-71 \text { años } \\
\text { NP }\end{array}$ & $\begin{array}{l}202 \text { hombres } \\
72 \text { mujeres }\end{array}$ & Perú & $\begin{array}{l}\text { El } 46.3 \% \text { de la muestra presenta } \\
\text { niveles significativos de SB }\end{array}$ \\
\hline
\end{tabular}

Nota. N/E: No Especifica, PRO: Muestreo probabilístico, NP: Muestreo no probabilístico, SB: Síndrome de Burnout.

Con relación al procedimiento, la recolección de tipo off line predominó en las publicaciones; el $88 \%$ proporcionó información del lugar en donde fueron aplicados los instrumentos y solo un $16 \%$ indicó los periodos en los que se llevó a cabo la recolección de datos; es importante destacar que más del 70\% no informaron sobre la confidencialidad y anonimato en la recolección, al igual que no se menciona Comité de ética o marcos legales y éticos para la aplicación de instrumentos; únicamente el 60\% utilizó el consentimiento informado (verbal o escrito) en el procedimiento (ver Tabla 3).

Tabla 3

Características del procedimiento de recolección y aspectos éticos.

\begin{tabular}{|c|c|c|c|c|c|c|}
\hline \multirow{2}{*}{ Autoría, año } & \multicolumn{3}{|c|}{ Recolección de datos } & \multicolumn{3}{|c|}{ Aspectos éticos } \\
\hline & Tipo & $\begin{array}{c}\text { Descripción } \\
\text { espacial }\end{array}$ & $\begin{array}{c}\text { Descripción } \\
\text { temporal }\end{array}$ & $\begin{array}{c}\text { Consentimiento } \\
\text { informado }\end{array}$ & $\begin{array}{c}\text { Comité de } \\
\text { ética }\end{array}$ & $\begin{array}{c}\text { Marco legal } \\
\text { y ético }\end{array}$ \\
\hline \multicolumn{7}{|l|}{ García-Arroyo } \\
\hline $\begin{array}{l}\text { \& Osca } \\
(2019)\end{array}$ & Off & Sí & No & - & - & - \\
\hline Romero, 2019 & Off & No & No & $\sqrt{ }$ & - & - \\
\hline $\begin{array}{l}\text { Villamar et al. } \\
\text { (2019) }\end{array}$ & On & Sí & No & $\sqrt{ }$ & - & - \\
\hline $\begin{array}{l}\text { Mesurado \& } \\
\text { Laudadío } \\
\text { (2019) }\end{array}$ & On & Sí & No & $\sqrt{ }$ & - & - \\
\hline Alves, 2019 & Off & No & No & $\sqrt{ }$ & $\sqrt{ }$ & - \\
\hline $\begin{array}{c}\text { Alpízar \& } \\
\text { Araya (2018) }\end{array}$ & On-Off & Sí & No & - & - & - \\
\hline \multicolumn{7}{|l|}{ Rodríguez \& } \\
\hline $\begin{array}{c}\text { Sánchez } \\
\text { (2018) }\end{array}$ & Off & Sí & No & $\sqrt{ }$ & - & - \\
\hline Brito, 2018 & Off line & Sí & No & - & - & - \\
\hline \multicolumn{7}{|l|}{ García-Arroyo } \\
\hline $\begin{array}{l}\text { \& Osca } \\
\text { (2017) }\end{array}$ & Off & Sí & No & - & - & - \\
\hline $\begin{array}{l}\text { García et al. } \\
\text { (2017) }\end{array}$ & Off & Sí & Sí & $\sqrt{ }$ & - & - \\
\hline $\begin{array}{l}\text { Jeunon et al. } \\
\text { (2017) }\end{array}$ & Off & Sí & No & - & - & - \\
\hline $\begin{array}{l}\text { Cabezas, } \\
2016\end{array}$ & - & No & No & - & - & - \\
\hline
\end{tabular}

Espiral. Cuadernos del Profesorado | ISSN 1988-7701 | 2021, 14(29), 136-150 


\begin{tabular}{|c|c|c|c|c|c|c|}
\hline $\begin{array}{l}\text { Bustamante et } \\
\text { al. (2016) }\end{array}$ & Off & Sí & No & - & - & - \\
\hline $\begin{array}{l}\text { Ilaja \& Reyes } \\
\text { (2016) }\end{array}$ & Off & Sí & Sí & - & - & - \\
\hline $\begin{array}{l}\text { Chavarría et } \\
\text { al. (2016) }\end{array}$ & Off & Sí & No & $\sqrt{ }$ & - & - \\
\hline $\begin{array}{l}\text { Ruiz et al. } \\
\text { (2015) }\end{array}$ & - & Sí & No & $\sqrt{ }$ & - & $\sqrt{ }$ \\
\hline $\begin{array}{l}\text { Cárdenas et al. } \\
\text { (2014) }\end{array}$ & Off & Sí & No & - & - & - \\
\hline $\begin{array}{l}\text { Gaxiola-Villa, } \\
2014\end{array}$ & Off & Sí & No & $\sqrt{ }$ & - & - \\
\hline $\begin{array}{l}\text { Zárate \& Arita } \\
\quad \text { (2013) }\end{array}$ & Off & Sí & Sí & - & - & - \\
\hline $\begin{array}{l}\text { Borges et al. } \\
\quad(2012)\end{array}$ & Off & Sí & No & $\sqrt{ }$ & - & - \\
\hline $\begin{array}{c}\text { Rojas \& } \\
\text { Grisales } \\
(2011)\end{array}$ & Off & Sí & Sí & $\sqrt{ }$ & $\sqrt{ }$ & - \\
\hline $\begin{array}{l}\text { Ferrel et al. } \\
\quad(2010)\end{array}$ & - & Sí & No & - & - & - \\
\hline $\begin{array}{l}\text { Caballero et } \\
\text { al. (2009) }\end{array}$ & Off & Sí & No & $\sqrt{ }$ & - & - \\
\hline $\begin{array}{l}\text { Rojas et al. } \\
\text { (2009) }\end{array}$ & Off & Sí & No & $\sqrt{ }$ & $\sqrt{ }$ & $\sqrt{ }$ \\
\hline $\begin{array}{l}\text { Ponce et al. } \\
(2005)\end{array}$ & - & Sí & No & - & - & - \\
\hline
\end{tabular}

Nota: Off: Investigación presencial, On: Investigación virtual, - - No especifica.

Finalmente, la última pregunta (¿Cuáles son los instrumentos de medida utilizados en las investigaciones?) se señaló que el MBI (Maslach \& Jackson, 1986) es la herramienta más utilizada en sus diferentes versiones (72\%), sin embargo, no se excluyen otras escalas como el CESQT (10\%), OLBI (8\%) o CBP-R (10\%) (ver Tabla 4).

Tabla 4

Instrumentos de evaluación.

\begin{tabular}{|c|c|c|}
\hline Autoría, año & Instrumento/ Año & $\begin{array}{l}\text { Escala (Ítems) } \\
\text { Dimensiones (Ítems) }\end{array}$ \\
\hline $\begin{array}{c}\text { García-Arroyo \& Osca } \\
\text { (2019) }\end{array}$ & $\begin{array}{c}\text { MBI-GS } \\
1996\end{array}$ & $\begin{array}{l}\text { Escala tridimensional (N/E) } \\
\text { *Agotamiento emocional (5) } \\
\text { Despersonalización } \\
\text { Realización personal }\end{array}$ \\
\hline Romero, 2019 & $\begin{array}{l}\text { QPW-5 } \\
\text { N/E }\end{array}$ & $\begin{array}{l}\text { Escala multidimensional }(127) \\
\text { *Condiciones de trabajo } \\
\quad \text { *Carga de trabajo } \\
\text { *Bienestar laboral general } \\
\text { *Inventario de Burnout de Maslach } \\
\quad \text { *Engagement }\end{array}$ \\
\hline Villamar et al. (2019) & $\begin{array}{l}\text { CESQT-PE } \\
2011\end{array}$ & $\begin{array}{l}\text { Escala multidimensional }(20) \\
\text { *Ilusión por el trabajo } \\
\text { *Desgaste psíquico } \\
\text { *Indolencia } \\
\text { *Culpa }\end{array}$ \\
\hline $\begin{array}{l}\text { Mesurado \& Laudadío } \\
\text { (2019) }\end{array}$ & $\begin{array}{l}\text { OLBI } \\
2010\end{array}$ & $\begin{array}{l}\text { Escala bidimensional (16) } \\
\quad \text { *Agotamiento } \\
\quad \text { *Falta de engagement }\end{array}$ \\
\hline
\end{tabular}

Espiral. Cuadernos del Profesorado | ISSN 1988-7701 | 2021, 14(29), 136-150 


\begin{tabular}{|c|c|c|}
\hline Alves, 2019 & $\begin{array}{l}\text { OLBI } \\
2003\end{array}$ & $\begin{array}{c}\text { Escala bidimensional (9) } \\
* \text { Disengagement }(6) \\
* \text { Agotamiento }(3)\end{array}$ \\
\hline \multirow[t]{4}{*}{ Alpízar \& Araya (2018) } & MBI & Escala tridimensional (22) \\
\hline & $\mathrm{N} / \mathrm{E}$ & *Agotamiento \\
\hline & & *Despersonalización \\
\hline & & $*$ Realización personal \\
\hline \multirow[t]{4}{*}{ Rodríguez \& Sánchez (2018) } & MBI & Escala tridimensional (22) \\
\hline & $1981 ; 1986$ & *Cansancio emocional (9) \\
\hline & & *Despersonalización (5) \\
\hline & & *Realización personal (8) \\
\hline \multirow[t]{4}{*}{ Brito, 2018} & MBI-GS & Escala tridimensional (15) \\
\hline & 1996 & *Agotamiento $(5)$ \\
\hline & & $*$ Cinismo $(4)$ \\
\hline & & *Eficacia profesional (6) \\
\hline \multirow{4}{*}{$\begin{array}{c}\text { García-Arroyo \& Osca } \\
\text { (2017) }\end{array}$} & MBI-GS & Escala tridimensional (16) \\
\hline & 1996 & *Agotamiento emocional (5) \\
\hline & & $*$ Cinismo $(5)$ \\
\hline & & *Realización profesional (6) \\
\hline \multirow[t]{4}{*}{ García et al. (2017) } & MBI & Escala tridimensional (22) \\
\hline & $1981 ; 1986$ & *Cansancio emocional \\
\hline & & *Despersonalización \\
\hline & & *Realización personal \\
\hline \multirow[t]{4}{*}{ Jeunon et al. (2017) } & MBI-ES & Escala tridimensional (22) \\
\hline & 1981 & *Agotamiento emocional (9) \\
\hline & & *Despersonalización (5) \\
\hline & & *Bajo rendimiento profesional (8) \\
\hline \multirow[t]{4}{*}{ Cabezas, 2016} & MBI & Escala tridimensional (22) \\
\hline & $\mathrm{N} / \mathrm{E}$ & *Cansancio emocional \\
\hline & & *Despersonalización \\
\hline & & *Realización Personal \\
\hline \multirow[t]{4}{*}{ Bustamante et al. (2016) } & MBI & Escala tridimensional (22) \\
\hline & 1986 & *Agotamiento $(9)$ \\
\hline & & *Despersonalización (5) \\
\hline & & *Logro personal (8) \\
\hline \multirow[t]{4}{*}{ Ilaja \& Reyes (2016) } & MBI-ES & Escala tridimensional (N/E) \\
\hline & 1981 & *Agotamiento emocional \\
\hline & & *Despersonalización \\
\hline & & *Baja realización personal en el trabajo \\
\hline \multirow[t]{5}{*}{ Chavarría et al. (2016) } & CBP-R & Escala multidimensional (66) \\
\hline & 2000 & *Estrés de rol \\
\hline & & *Burnout \\
\hline & & *Desorganización institucional \\
\hline & & *Problemática administrativa \\
\hline \multirow[t]{4}{*}{ Ruiz et al. (2015) } & MBI & Escala tridimensional (22) \\
\hline & 1986 & *Cansancio emocional \\
\hline & & *Despersonalización \\
\hline & & *Baja realización personal \\
\hline \multirow[t]{5}{*}{ Cárdenas et al. (2014) } & CBP-R & Escala multidimensional (66) \\
\hline & 2000 & *Estrés de rol \\
\hline & & *Burnout \\
\hline & & *Desorganización institucional \\
\hline & & *Problemática administrativa \\
\hline \multirow[t]{4}{*}{ Gaxiola-Villa, 2014} & MBI & Escala tridimensional (N/E) \\
\hline & 1986 & *Agotamiento emocional \\
\hline & & Despersonalización \\
\hline & & *Baja realización personal \\
\hline Zárate \& Arita (2013) & CESQT & Escala multidimensional (28) \\
\hline
\end{tabular}




\begin{tabular}{|c|c|c|}
\hline & 2005 & $\begin{array}{l}\text { *Ilusión por el trabajo } \\
\text { *Desgaste psíquico } \\
\text { *Indolencia } \\
\text { *Culpa } \\
\text { *Desencanto profesional }\end{array}$ \\
\hline Borges et al. (2012) & $\begin{array}{c}\text { MBI-HSS } \\
1986\end{array}$ & $\begin{array}{l}\text { Escala tridimensional }(22) \\
\text { *Agotamiento emocional } \\
\text { *Despersonalización } \\
\text { *Falta de realización personal }\end{array}$ \\
\hline Rojas \& Grisales (2011) & $\begin{array}{l}\text { MBI } \\
2001\end{array}$ & $\begin{array}{l}\text { Escala tridimensional }(22) \\
\quad * \text { Fatiga emocional }(9) \\
\quad \text { *Despersonalización }(5) \\
\quad \text { *Bajo rendimiento personal (8) }\end{array}$ \\
\hline Ferrel et al. (2010) & $\begin{array}{c}\text { MBI-ED } \\
1986\end{array}$ & $\begin{array}{l}\text { Escala tridimensional }(22) \\
\text { *Cansancio emocional } \\
\text { *Despersonalización } \\
\text { *Realización personal }\end{array}$ \\
\hline Caballero et al. (2009) & $\begin{array}{c}\text { CESQT } \\
2003\end{array}$ & $\begin{array}{l}\text { Escala multidimensional (20) } \\
\text { *Desgaste psíquico (4) } \\
\text { *Indolencia (6) } \\
\text { *Ilusión por el trabajo (5) } \\
\text { Culpa (5) }\end{array}$ \\
\hline & $\begin{array}{c}\text { CBP-R } \\
2000\end{array}$ & $\begin{array}{l}\text { Escala multidimensional (66) } \\
\text { Factores psicosociales: } \\
\text { *Preocupaciones profesionales (7) } \\
\text { *Falta de reconocimiento profesional (4) }\end{array}$ \\
\hline Rojas et al. (2009) & $\begin{array}{l}\text { MBI } \\
2001\end{array}$ & $\begin{array}{l}\text { Escala tridimensional }(22) \\
\text { *Cansancio emocional } \\
\text { *Despersonalización } \\
\text { *Baja realización personal }\end{array}$ \\
\hline Ponce et al. (2005) & $\begin{array}{l}\text { MBI } \\
1986\end{array}$ & $\begin{array}{l}\text { Escala tridimensional }(22) \\
\text { *Cansancio emocional (9) } \\
\text { *Despersonalización }(5) \\
\text { *Realización personal }(8)\end{array}$ \\
\hline
\end{tabular}

Nota. *:Dimensión implementada, N/E: No específica, MBI: Maslach Burnout Inventory, MBI-GS: Maslach Burnout Inventory-General Survey, CESQT-PE: Cuestionario para la Evaluación del Síndrome de Quemarse por el TrabajoProfesionales de la Educación, OLBI: Oldenburg Burnout Inventory, MBI-ED: Maslach Burnout Inventory-Educadores, MBIES: Maslach Burnout Inventory-Educators Survey, CBP-R: Cuestionario de Burnout del Profesorado-Revisado, CESQT: Cuestionario para la Evaluación del Síndrome de Quemarse por el Trabajo, MBI-HSS: Maslach Burnout Inventory-Human Services Survey.

\section{Discusión}

En la presente revisión se visualiza el desarrollo de la investigación sobre SB en docentes universitarios latinoamericanos, realizada durante las dos últimas décadas. Los datos obtenidos apuntan a una gran presencia de producción científica en español, evidenciando un interés progresivo a partir del 2017. Asimismo, resaltó la variedad de palabras clave para el constructo en ambos idiomas (Quiceno \& Vinaccia, 2007), implicando confusiones en las traducciones o términos vinculados, por ejemplo, con el estrés laboral el cual algunos autores sugieren que es homólogo al burnout (Pérez, 2018), empero la evidencia empírica acumulada ha señalado una diferencia entre ambos fenómenos (Maslach, 2009).

Ahora bien, predominó el enfoque cuantitativo, así como el diseño de estudio no experimental con corte transversal por lo que el uso de esta metodología puede entenderse si los propósitos de las investigaciones son locales, descriptivas y, ulteriormente, diagnósticas; no obstante, implica limitaciones explicativas del fenómeno a través del tiempo, haciendo necesarios estudios longitudinales (García, 2004) sin olvidar la pertinencia de enfoques comprensivos, es decir cualitativos o mixtos (Flores-Kanter \& Medrano, 2019; Moscoso, 2017). 
Concerniente al muestreo, la mayoría de las investigaciones utilizaron una selección no probabilística la cual conlleva desventajas metodológicas, debido a que este tipo de muestreos se consideran informales, tienden a sesgarse y no ser representativos, de igual manera, los hallazgos son difícilmente generalizables en la población (Argibay, 2009; Pimienta, 2000), todo lo anterior en detrimento de la reproducibilidad de los resultados o del estudio (Maldonado, 2018). Sin embargo, es preciso reconocer su mérito y aportación, especialmente cuando se dificulta la accesibilidad a una muestra o grupo en específico, sin olvidar que el coste y la inversión de tiempo es menor a diferencia de un estudio probabilístico (Figueroa et al., 2019).

En ese mismo orden de ideas, las muestras de estudio se conformaron por ambos sexos, sin embargo, se halló que los hombres tuvieron una mayor participación, salvo en el caso de Gaxiola-Villa (2014) que utilizó exclusivamente población femenina, al respecto sería conveniente subsanar estos desequilibrios utilizando muestras homogéneas para tener un panorama completo del fenómeno. Por otro lado, es conveniente señalar que la mayor parte de los artículos analizados incluyeron muestra de docentes colombianos, lo que permite corroborar el interés sobre el tema en ese país y que ya ha sido apuntado anteriormente por otros autores (Casallas et al., 2017; Chávez, 2016).

Sobre la modalidad de recolección de datos, se encontró que la mayoría fue vía off line; al respecto es preciso señalar que poco a poco hay más estudios on line, se trata de una práctica que se ha empleado con mayor frecuencia en la investigación social y psicológica (Walther, 2002), tal vez por las muchas ventajas que supone como la reducción de costes, la facilitad de la recogida de datos en muestras grandes y dispersas, la disminución de la deseabilidad social en los participantes y el amplio acceso a la población (Abundis, 2016); no obstante, es importante recordar algunas de las dificultades asociadas a este tipo de metodología, como por ejemplo los posibles sesgos muestrales, así como algunos retos éticos, técnicos o metodológicos en su práctica (Eiorá et al., 2008; Tejada, 2018).

Con respecto al procedimiento y la aplicación de instrumentos sería ideal que previamente existiera un respaldo por parte de un Comité de ética con el fin de salvaguardar los principios éticos de la investigación científica, empero es conveniente apuntar el carácter relativamente reciente de la necesidad generalizada de la aprobación a proyectos de esta naturaleza, lo que no obsta para subrayar su carácter deseable. Del mismo modo, es fundamental usar oportunamente el consentimiento informado el cual no solo implica velar por el bienestar del individuo sino considerar su libre determinación y autonomía para su participación (Chávez et al., 2013).

Con relación a los instrumentos, sobresale el uso de la primera (Maslach \& Jackson, 1981) y segunda edición (Maslach \& Jackson, 1986) del MBI, el cual es una de las herramientas más utilizadas por los autores en sus diferentes versiones (León, 2009), esto podría deberse a que es considerada como una escala útil para evaluar al fenómeno en múltiples ocupaciones laborales y, por ello, ha obtenido reconocimiento dentro de la comunidad científica (Lubbadeh, 2020); no obstante, se han señalado algunas limitaciones en su contenido (Demerouti et al., 2010), comercialización y, por ende, en la replicación de resultados en otras zonas y regiones (Gil-Monte et al., 2009).

En lo que concierne a las limitaciones del presente estudio, destaca en primer lugar su carácter exploratorio, así como los criterios de inclusión implementados que pudieron excluir pesquisas de interés. Sin embargo, es importante subrayar que la principal limitación estriba en la inclusión únicamente de estudios no experimentales en la muestra, razón por la cual es preciso tener cautela en las inferencias obtenidas a la luz de las amenazas específicas a la validez interna y externa a este tipo de diseños, en general, y, en particular, de los trabajos incluidos. Es por ello que, además, no se pudo realizar un análisis de la calidad metodológica ni del riesgo de sesgo de los trabajos no experimentales revisados, aspectos que son más propios de diseños manipulativos. Pese a lo anterior, es posible destacar como fortaleza del presente estudio que se trata de la segunda revisión sistemática sobre síndrome de Burnout en docentes universitarios latinoamericanos, al menos al momento de la realización del manuscrito, actualizando parte de lo hallado en la revisión sistemática realizada por Chávez (2016), en especial en la prevalencia del fenómeno y el uso frecuente del MBI como instrumento para evaluar el SB, y agregando como novedad el análisis metodológico y ético de las publicaciones incluidas. 
Ahora bien, futuras investigaciones podrían analizar al SB en función de distintas variables sociodemográficas (i. e. sexo, edad), la implicación de factores asociados de índole personal (i.e. ansiedad, depresión, estrés laboral), organizacionales y sociales. De igual modo, sería pertinente realizar estudios longitudinales (Rodríguez et al., 2019) o experimentales, que permitan el seguimiento del fenómeno a lo largo del tiempo o identificar el vínculo causal entre dichas variables, del mismo modo que se requieren contribuciones enfocadas sobre factores protectores que ayudarían al bienestar de las y los docentes ante el burnout. Todo ello sin olvidar la importancia de contextualizar teórica y metodológicamente las condiciones actuales en las que se realiza la labor docente, por ejemplo, la relación entre la reclusión debido a la pandemia, el teletrabajo y el estrés.

\section{Conclusiones}

A manera de conclusión, con los resultados obtenidos en esta revisión exploratoria se evidenció una pluralidad en la producción científica perteneciente al territorio latinoamericano, esto supone que la población docente universitaria es vulnerable ante dicho fenómeno (Rodríguez-García et al., 2017); de igual modo se distingue una gran diversidad de metodologías, instrumentos y procedimientos implementados en las pesquisas lo que confirma que el SB es un objeto de estudio en el ámbito laboral que incluye a la docencia (Dalagasperina \& Monteiro, 2014).

Ahora bien, en cuanto a las posibles implicaciones derivadas de esta revisión podría destacarse que: 1) en lo preventivo sería pertinente una mayor comprensión de las demandas emocionales, su influencia en el alumnado, así como las posibles estrategias de afrontamiento; lo cual, a su vez, derivaría en una gran necesidad de ampliar el conocimiento de los factores protectores y su función ante el SB en aras no solo de prevenir su aparición sino en aminorar sus efectos; en ese sentido, 2) en los aspectos de intervención, es preciso recordar que la mayoría de las investigaciones analizadas exploraron las causas del burnout, resaltando así la ausencia de planes o programas de intervención, tal vez por el carácter aún incipiente del estudio del fenómeno en la región o por el bajo o nulo interés sobre el problema por parte de instituciones y autoridades educativas. En ese orden de ideas es importante recordar el papel que las políticas públicas tienen en la orientación y regulación del trabajo del cuerpo docente de cualquier nivel educativo, en especial se hace muy importante su adaptación a las actuales condiciones de teletrabajo donde el estrés y el burnout pueden eclosionar aun con más fuerza.

Por otro lado, dentro de las implicaciones 1) empíricas se hace necesario un mayor número de estudios que favorezca el desarrollo de un corpus teórico y empírico donde se delimite claramente el concepto del estrés con el de burnout; 2) en lo metodológico, se requiere de una mayor inclusión de participantes mujeres con el objetivo de visibilizar el fenómeno desde una perspectiva de género; así mismo, se debería tener en cuenta a las características de la muestra con especial énfasis en las necesidades específicas de los participantes y el contexto situacional en el que ejercen sus labores; por ende, sería importante prestar una mayor atención a la modalidad virtual en las que actualmente ejercen sus labores docentes debido a la contingencia sanitaria. Finalmente, 3) en lo instrumental, sería idónea la creación o diversificación de escalas sobre SB contextualizadas a las poblaciones de estudio sin olvidar la validación de dichos instrumentos.

Contribución de cada Autor: "conceptualización, J.L.R-S., B.A.T-R. y D.J.R-V.; metodología, J.L.R-S., B.A.TR. y D.J.R-V; análisis, J.L.R-S., B.A.T-R.; escritura del manuscrito, J.L.R-S., B.A.T-R.; escritura, revisión y edición J.L.R-S. y D.J.R-V; supervisión, J.L.R-S.”

Financiación: Esta investigación no recibió ninguna financiación externa.

Conflicto de Intereses: Los autores declaran que no tienen conflicto de intereses.

\section{Referencias}

*Artículos incluidos en la revisión sistemática

Abundis, V. M. (2016). Beneficios de las encuestas electrónicas como apoyo para la investigación. Tlatemoani, (22), 168-186. https://bit.ly/3hpH39v

American Psychological Association (APA). (2017). Ethical Principles of Psychologists and Code of Conduct. Autor.

Espiral. Cuadernos del Profesorado | ISSN 1988-7701 | 2021, 14(29), 136-150 
*Alpízar, H. Y., \& Araya, C. (2018). Diagnóstico del síndrome de burnout en docentes universitarios por medio del modelo de clases latentes: una aplicación del Bootstrap Paramétrico. Revista Pensamiento Actual, 18(31), 24-34. https://bit.ly/3drLOgr

*Alves, P. C., Oliviera, A. D. F., \& Paro, H. B. M. D. S. (2019). Quality of life and burnout among faculty members: How much does the field of knowledge matter? PloS ONE, 14(3), 1-12. https://doi.org/10.1371/journal.pone.0214217

Argibay, J. C. (2009). Muestra en investigación cuantitativa. Subjetividad y procesos cognitivos, 13(1), 13-29. https://doi.org/10.15517/PA.V18I31.35669

*Borges, A., Ruiz, M., Rangel, R., \& González, P. (2012). Síndrome de Burnout en docentes de una universidad pública venezolana. Comunidad y Salud, 10(1), 1-9. https://bit.ly/2M3TPNz

*Brito, J. (2018). Calidad educativa en las instituciones de educación superior: evaluación del síndrome de burnout en los profesores. Revista Iberoamericana para la Investigación y el Desarrollo Educativo, 8(16), 1-19. https://bit.ly/3duzD2z

*Bustamante, E., Bustamante, F., González, G., \& Bustamante, L. (2016). El burnout en la profesión docente: un estudio en la escuela de bioanálisis de la Universidad de Carabobo Sede Aragua, Venezuela. Medicina y Seguridad del trabajo, 62(243), 111-121. https://bit.ly/3pzCgmf

*Caballero, C., González, O., Mercado, D., Llanos, C., Bermejo, Y., \& Vergel, C. (2009). Prevalencia del síndrome de burnout y su correlación con factores psicosociales en docentes de una institución universitaria privada de la ciudad de Barranquilla. Psicogente, 12(21), 142-157. https://bit.ly/3pxvbTg

*Cabezas, E. (2016). Análisis del síndrome de Burnout en docentes de la Facultad de Ingeniería de la Universidad Nacional de Chimborazo y su incidencia en el desempeño laboral. Producción y Gestión, 19(1), 59-68. https://bit.ly/2ZAi5tz

*Cárdenas, M., Méndez, L. M., \& González, M. T. (2014). Evaluación del desempeño docente, estrés y burnout en profesores universitarios. Actualidades Investigativas en Educación, 14(1), 1-22. https://bit.ly/3aw0yZP

Casallas, J. A., Rodríguez, A. P., \& Ladino, A. J. (2017). Revisión teórica: el estado actual de las investigaciones sobre el síndrome de burnout en docentes de Latinoamérica de habla hispana, entre los años 2010 al 2017 (Tesis de Licenciatura). https://bit.ly/3bmDBaG

*Chavarría, R. A., Colunga, F. J., Loria, J., \& Peláez, M. (2016). Síndrome de burnout en médicos docentes de un hospital de 2. ${ }^{\circ}$ nivel en México. Educación Médica, 18(4), 254-261. http://dx.doi.org/10.1016/j.edumed.2016.09.001

Chávez, G., Cruz, H. S., \& Grimaldo, M. P. (2013). El consentimiento informado en las publicaciones latinoamericanas de psicología. Avances en Psicología Latinoamericana, 32(2), 345-359. https://doi.org/10.12804/apl32.2.2014.12

Chávez, C. A. (2016). Síndrome de Burnout en docentes universitarios. INNOVA Research Journal, 1(9), 77-95. https://doi.org/10.33890/innova.v1.n9.2016.55

Dalagasperina, P., \& Monteiro, J. K. (2014). Preditores da síndrome de burnout em docentes do ensino privado. Psico-USF, 19(2), 263-275. https://doi.org/10.1590/1413-82712014019002011

Demerouti, E., Mostert, K., \& Bakker, A.B. (2010). Burnout and work engagement: A thorough investigation of the independency of both constructs. Journal of Occupational Health Psychology, 15(3), 209-222. https://doi.org/10.1037/a0019408

Eiorá, F. J., Fernández, I., \& Pérez, P. (2008). Cuestionarios psicológicos e investigación en Internet: Una revisión de la literatura. Anales de Psicología, 24(1), 150-157. https://bit.ly/37rg7Qy

Fernández-Sánchez, H., King, K., \& Enríquez-Hernández, C. B. (2020). Revisiones Sistemáticas Exploratorias como metodología para la síntesis del conocimiento científico. Enfermería Universitaria, 17(1), 87-94. https://doi.org/10.22201/eneo.23958421e.2020.1.697

*Ferrel, R., Pedraza, C., \& Rubio, B. (2010). El síndrome de quemarse en el trabajo (burnout) en docentes universitarios. Duazary, 7(1), 15-28. https://doi.org/10.21676/2389783X.306

Figueroa, M., Borja, E., Plúas, R. M., Castro, G. J., Tovar, G. R., \& Estupiñan, J. (2019). Estudio situacional para determinar estrategias formativas en la atención a escolares con necesidades educativas especiales en la zona 5 del Ecuador. Revista Investigación Operacional, 40(2), 255-266. https://bit.ly/2M7TMAl 
Flores-Kanter, P. E., \& Medrano, L. A. (2019). Núcleo básico en el análisis de datos cualitativos: Pasos, técnicas de identificación de temas y formas de presentación de resultados. Interdisciplinaria Revista de Psicología y Ciencias Afines, 36(2), 203-215. https://bit.ly/3bj60P5

*García-Arroyo, J. A., \& Osca, A. (2017). Coping with burnout: Analysis of linear, non-linear and interaction relationships. Anales de psicología, 33(3), 722-731. http://dx.doi.org/10.6018/analesps.33.3.279441

*García-Arroyo, J. A., \& Osca, A. (2019). Work Overload and Emotional Exhaustion in University Teachers: Moderating Effects of Coping Styles. Universitas Psychologica, 18(2), 1-12. https://doi.org/10.11144/Javeriana.upsy18-2.woee

*García, A. A., Escorcia, C. V., \& Perez, B. S. (2017). Síndrome de Burnout y sentimiento de autoeficacia en profesores universitarios. Propósitos y Representaciones, 5(2), 65-126. http://dx.doi.org/10.20511/pyr2017.v5n2.170

García, J. (2004). Estudios descriptivos. Nure Investigación, 7, 1-3. https://bit.ly/2ZqZGzD

*Gaxiola-Villa, E. (2014). Bienestar psicológico y desgaste profesional [DP] en amas de casa docentes universitarias. Journal of Behavior, Health \& Social Issues, 6(1), 79-88. https://doi.org/10.5460/jbhsi.v6.1.47605

Gil-Monte, P. R., Unda, S., \& Sandoval, J. I. (2009). Validez factorial del «Cuestionario para la Evaluación del Síndrome de Quemarse por el Trabajo» (CESQT) en una muestra de maestros mexicanos. Salud Mental, 32(3), 205-214. https://bit.ly/3prD2BG

*Ilaja, B., \& Reyes, C. (2016). Burnout y estrategias de inteligencia emocional en profesores universitarios: implicaciones en la salud laboral educativa. Psicología desde el Caribe, 33(1), 31-46. http://dx.doi.org/10.14482/psdc.33.1.8081

Juárez-García, A., Idrovo, Á. J., Camacho-Ávila, A., \& Placencia-Reyes, O. (2014). Síndrome de burnout en población mexicana: Una revisión sistemática. Salud Mental, 37(2), 159-176. https://bit.ly/37tZhAG

*Jeunon, E. E., Correa. L. Á., Duarte, L., \& Guimaraes, E. H. (2017). Burnout Syndrome: a study with professors at a public university in Maranhao. Revista Gestao \& Tecnologia, 17(2), 252-268. https://doi:10.20397/g\&t

Lackritz, J. R. (2004). Exploring burnout among university faculty: incidence, performance, and demographic issues. Teaching and Teacher Education, 20(7), 713-729. https://doi:10.1016/j.tate.2004.07.002

León, E. F. (2009). Angustia docente: una revisión de la investigación del malestar y la violencia docente en Latinoamérica. Innovar, Especial en Educación, 19, 91-110. https://bit.ly/37xDsQL

Lubbadeh, T. (2020). Job burnout: a general literature review. International Review of Management and Marketing, 10(3), 7-15. https://doi.org/10.32479/irmm.9398

*Mesurado, B., \& Laudadío, J. (2019). Experiencia profesional, capital psicológico y engagement. Su relación con el burnout en docentes universitarios. Propósitos y Representaciones, 7(3), 12-40. https://doi:10.20511/pyr2019.v7n3.327

Maldonado, J. (2018). Metodología de la Investigación social: Paradigmas: cuantitativo, sociocrítico, cualitativo, complementario. Ediciones de la U. https://bit.ly/3bqMSP0

Maslach, C., \& Jackson, S. E. (1981). MBI: Maslach Burnout Inventory Manual. Consulting Psychologists Press.

Maslach, C., \& Jackson, S. E. (1986). Maslach Burnout Inventory Manual (2.a ed.). Consulting Psychologist Press.

Maslach, C. (2009). Comprendiendo el burnout. Ciencia \& Trabajo, 11(32), 37-43.

Moher, D., Liberati, A., Tetzlaff, J., Altman, D., \& The PRISMA Group. (2009). Preferred Reporting Items for Systematic Reviews and MetaAnalyses: The PRISMA Statement. PLoS Medicine, 6(6), e1000097. https://doi:10.1371/journal.pmed1000097

Moscoso, J. (2017). Los métodos mixtos en la investigación en educación: hacia un uso reflexivo. Cadernos de Pesquisa, 47(164), 632-649. https://doi:10.1590/198053143763

Palmer, L. Y., Prince, R., Medina, C., Figueroa, M., López, R., \& Rodríguez, G. (2016). Prevalencia del síndrome de Burnout en docentes de la Universidad Autónoma de Baja California, Mexicali, México. Revista Cubana de Salud y Trabajo, 17(3), 36-40. https://bit.ly/3k38TYp

Pérez, I. (2018). Síndrome de Burnout, contexto social y subjetividad. Revista de Ciencias Empresariales y Sociales, 1(1), 107-136. https://bit.ly/3dCyMNc

Pimienta, R. (2000). Encuestas probabilísticas vs. no probabilísticas. Política y cultura, (13), 263-276. https://bit.ly/2MbloWO 
*Ponce, C. R., Bulnes, M. S., Aliaga, J. R., Atalaya, M. C., \& Huertas, R. E. (2005). El síndrome del quemado por estrés laboral asistencial en grupos de docentes universitarios. Revista IIPSI, 8(2), 87-112. https://doi:10.15381/rinvp.v8i2.4050

Pujol-Cols, L., Foutel, M., \& Porta, L. (2019). Riesgos psicosociales en la profesión académica: un análisis interpretativo del discurso de docentes universitarios argentinos. Trabajo y Sociedad, (33), 197-223. https://bit.ly/3pAiHKr

Quiceno, J. M., \& Vinaccia, S. (2007). Burnout: "Síndrome de Quemarse en el trabajo (SQT)". Acta Colombiana de Psicología, 10(2), 117-125. https://bit.ly/3bqwoGT

*Rodríguez, E. A., \& Sánchez, M. (2018). Síndrome de Burnout y variables sociodemográficas en docentes de una Universidad privada de Lima. Revista de Investigación Educativa, 36(2), 401-419. https://doi:10.6018/rie.36.2.282661

Rodríguez-García, A.M., Sola-Martínez, T., \& Fernández-Cruz, M. (2017). Impacto del Burnout en el desarrollo profesional del profesorado universitario. Una revisión de la investigación. Revista Electrónica Interuniversitaria de Formación del Profesorado, 20(3), 161-178. https://doi:10.6018/reifop.20.3.275121

Rodríguez, I., Kozusznik, M. W., Peiró, J. M., \& Tordera, N. (2019). Individual, co-active and collective coping and organizational stress: A longitudinal study. European Management Journal, 37(1), 86-98. https://bit.ly/3dw3hES

*Rojas, M. L., \& Grisales, H. (2011). Burnout syndrome in professors from an academic unit of a Colombian university. Investigación y Educación en Enfermería, 29(3), 427-434. https://bit.ly/37x98pl

*Rojas, M. L., Zapata, J. A., \& Grisales, H. (2009). Síndrome de burnout y satisfacción laboral en docentes de una institución de educación superior, Medellín, 2008. Revista Facultad Nacional de Salud Pública, 27(2), 198210. https://bit.ly/3aCobjO

*Romero, M. P. (2019). Working conditions and the meaning of the academic profession in Colombian university professors. Acta Colombiana de Psicología, 22(2), 267-279. https://doi:10.14718/ACP.2019.22.2.13

*Ruiz, G., Baza, D., Rosa, G., Rodríguez, V., \& Vivic, K. (2015). Síndrome de Burnout en docentes universitarios. Revista Cubana de Enfermería, 31. https://bit.ly/2NLdp1j

Tejada, C. (2018). Comparación entre la aplicación en formato papel y en formato online de dos instrumentos de evaluación: Cuestionario de la Forma Corporal y Cuestionario de los Tres Factores versión revisada 18. Intervención en contextos clínicos y de la salud, 2, 271-278.

Urrútia, G., \& Bonfill, X. (2010). Declaración PRISMA: una propuesta para mejorar la publicación de revisiones sistemáticas y metaanálisis. Medicina Clínica, 135(11), 507-511. http://doi.org/10.1016/j.medcli.2010.01.015.

*Villamar, D., Juárez, A., González, I. G., \& Osnaya, M. (2019). Factores psicosociales y síndrome de Burnout en académicos de una universidad pública de México. Propósitos y Representaciones, 7(3), 111-126. https://doi:10.20511/pyr2019.v7n3.360

Walther, J. B. (2002). Research ethics in Internet-enabled research: Human subjects issues and methodological myopia. Ethics and Information Technology, 4(3), 205-216. https://doi:10.1023/a:1021368426115

*Zárate, N. E., \& Arita, B. Y. (2013). Condiciones predisponentes del Síndrome de Quemarse por el trabajo en odontólogos que ejercen la docencia. Oral, 14(2), 1056-1061. https://bit.ly/3qESSKL 